\title{
Biomimetic patterned surfaces for controllable friction in micro- and nanoscale devices
}

\author{
Arvind Singh ${ }^{1 *}$ and Kahp-Yang Suh ${ }^{2}$
}

\begin{abstract}
Biomimetics is the study and simulation of biological systems for desired functional properties. It involves the transformation of underlying principles discovered in nature into man-made technologies. In this context, natural surfaces have significantly inspired and motivated new solutions for micro- and nano-scale devices (e.g., Micro/ Nano-Electro-Mechanical Systems, MEMS/NEMS) towards controllable friction, during their operation. As a generic solution to reduce friction at small scale, various thin films/coatings have been employed in the last few decades. In recent years, inspiration from 'Lotus Effect' has initiated a new research direction for controllable friction with biomimetic patterned surfaces. By exploiting the intrinsic hydrophobicity and ability to reduce contact area, such micro- or nano-patterned surfaces have demonstrated great strength and potential for applications in MEMS/NEMS devices. This review highlights recent advancements on the design, development and performance of these biomimetic patterned surfaces. Also, we present some hybrid approaches to tackle current challenges in biomimetic tribological applications for MEMS/NEMS devices.
\end{abstract}

Keywords: Biomimetics; MEMS/NEMS; Silicon; Polymer; Friction; Wear; Lithography; Patterns; Contact area; Lubrication

\section{Introduction}

MEMS/NEMS are miniaturized devices that are built at micro/nano-scale and are operated by various electrical, mechanical, and optical principles. These include a variety of sensors and actuators for industrial, consumer, defense, aerospace, and biomedical applications [1]. In particular, actuator-based devices have elements that undergo relative mechanical motion such as microgears, micro-motors and micro-engines (e.g. Figure 1 [2]). In these devices, the issues of smooth operation and long-term reliability have remained un-resolved owing to high friction that arises at micro/nano-scale [3]. Due in part to this reason actuator-based devices have not been fully commercialized yet.

In general, lubrication is the most promising solution to reduce friction. However, lubrication becomes difficult when the dimensions of machine elements decrease from macro- to micro/nano-scale. For example, as the size of the machine elements shrinks from $1 \mathrm{~mm}$

\footnotetext{
* Correspondence: arvindsingh@ntu.edu.sg

${ }^{1}$ Energy Research Institute (ERIAN), Clean Tech One, Nanyang Technological University, Singapore 637141, Singapore

Full list of author information is available at the end of the article
}

to $1 \mu \mathrm{m}$, the surface area decreases by a factor of $10^{6}$ whereas the volume decreases by a factor of $10^{9}$, yielding the surface area-to-volume ratio of $10^{3}$. Hence, surface forces such as adhesion and friction become significantly influential at such small scales. Further in miniaturized devices, the gap (clearance) between the elements is small $(<1 \mu \mathrm{m})$, which prohibits the use of conventional liquid and/or solid lubricants. For example, a drop of liquid lubricant will be in millimeter size and the size of solid lubricants such as graphite or $\mathrm{MoS}_{2}$ is of the same order as the size of the miniaturized devices. In addition, retention of lubricants at the contact points between the moving elements could be a hurdle for further implementation. Thus, minimizing surface forces in miniaturized devices has remained a real challenge.

At micro/nano-scale, friction is in a regime where the contribution from intrinsic/inherent adhesion outweighs that from asperity deformation [4]. Therefore, friction becomes naturally reduced while minimizing intrinsic adhesion, which arises due to the contribution of various attractive forces such as capillary, electrostatic, van der Waal, and chemical forces under different circumstances [4]. Amongst these forces, the capillary force that causes 

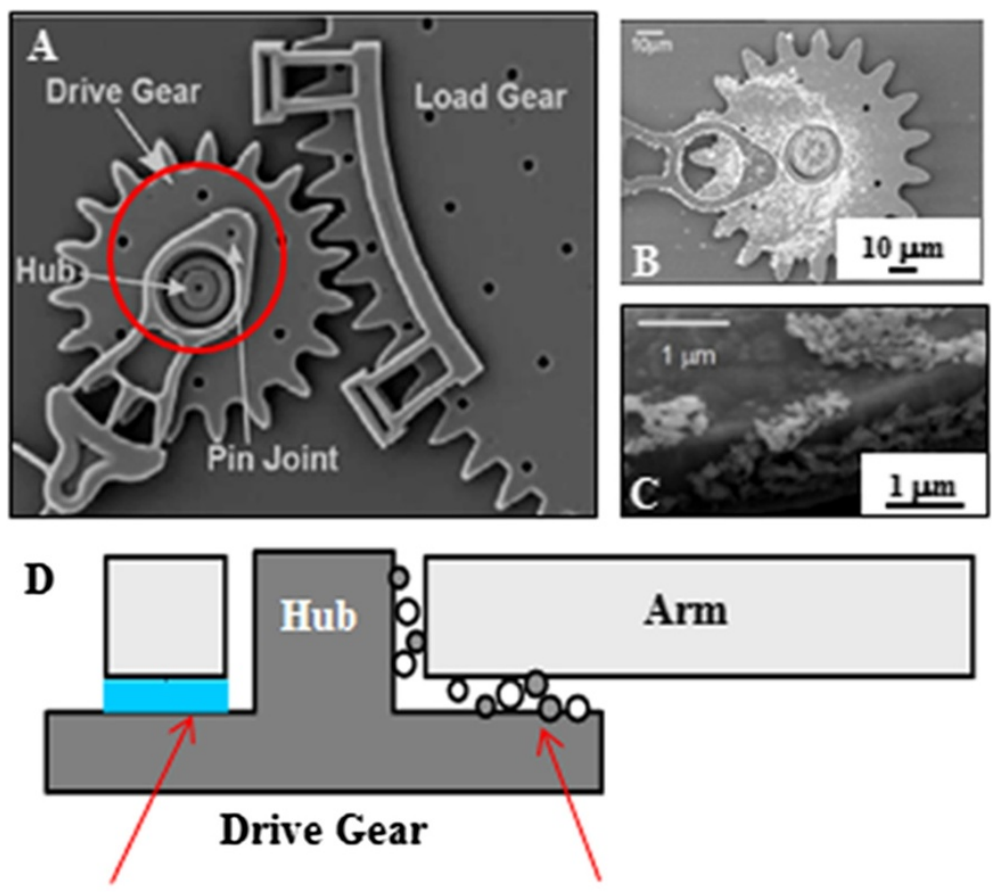

\section{Capillary Effect}

\section{Wear Particles}

Figure 1 Silicon MEMS [2,4,6]: (A) micro-engine gears, (B \& C) wear particles and (D) schematic of formation of meniscus bridge and wear particles.

the formation of meniscus-bridge arising from the condensation of water from the environment is strongest [4]. From a material point of view, silicon is the widely used material for the fabrication of MEMS/NEMS, and in recent years SU-8 polymer has become an attractive material [5]. Silicon is hydrophilic in nature and generates a strong capillary force, resulting in high friction that opposes easy motion of components (Figure 1) $[4,6]$. Silicon is also brittle and undergoes wear (Figure 1) $[2,6]$. In contrast, SU-8 polymer is hydrophobic, but exhibits high friction/wear due to its soft nature [7].

In the past, various chemical modifications of surfaces such as boundary lubricant thin films/coatings have been adopted to reduce intrinsic adhesion and friction by increasing hydrophobicity and lubricity of MEMS/NEMS materials. Examples include self-assembled monolayers (SAMs) [8], diamond-like carbon (DLC) coatings [9], and also vapor phase lubricants [4]. In order to overcome some of the limitations of chemical modifications, bio-inspired topographical modification of surfaces has recently emerged as an effective route to minimize friction at micro/nano-scale. Biomimetic patterned surfaces lower intrinsic adhesion and friction via hydrophobicity (i.e. reduction in surface energy) and decrease in physical contact area between surfaces. The biomimetic surfaces, when applied with nano-scale boundary lubricants, exhibit even more remarkable capability to reduce surface forces, as well as to enhance durability, i.e. wear resistance.

Recognizing this important trend in tribological applications, this review gives an overview on the design, development and performance of biomimetic patterned surfaces that can significantly control friction at micro/ nano-scale for applications in MEMS/NEMS. As an introduction, Table 1 presents the data of water contact angle (CA) and friction properties of various biomimetic patterned surfaces reported in the literature.

\section{Inspiration from Lotus Effect}

'Lotus Effect' refers to the extremely high water repellence and self-cleaning behavior exhibited by the leaves of lotus plant (Figure 2A). The surface of lotus leaves has tiny protuberances (15-20 $\mu \mathrm{m}$ high, $20-25 \mu \mathrm{m}$ apart) covered by waxy, hydrophobic crystals ( $1 \mathrm{~nm}$ in diameter) (Figure 2B) [10]. These features make the plant leaves superhydrophobic (water contact angle: $162^{\circ}$, contact angle hysteresis of $\left.4^{\circ} \pm 2^{\circ}[10,11]\right)$. Such an effect arises from heterogeneous/composite wetting of water, wherein water droplets sit on top of the protuberances with air trapped in between them, thus significantly reducing the solid contact area with the droplets [12]. The fact that the protuberances and wax on lotus leaves reduce surface energy and contact area is highly inspirational for controlling friction at small-scale. Hence, researchers have created 
Table 1 Water contact angle (CA) values, and friction properties of various biomimetic patterned surfaces at nano- and micro-scales

\begin{tabular}{|c|c|c|c|c|}
\hline Surface & CA (deg) & Nano-scale friction force $(\mathrm{nN})$ & Micro-scale friction coefficient $(\mu)$ & Ref \\
\hline Silicon flat surface & 22 & $20-28$ & 0.46 & [15] \\
\hline SU-8 thin film & 83 & - & 0.6 & [30] \\
\hline PMMA nano-patterns & 99 & $0.84-1.97$ & 0.33 & [13] \\
\hline DLC nano-dot surfaces & 95 & $0.98-1.63$ & 0.13 & [23] \\
\hline PMMA micro-patterns (Replicated Surfaces) & $91-106$ & - & $0.11-0.13$ & {$[25,26]$} \\
\hline Silicon micro-pillars & 59 & - & 0.16 & [29] \\
\hline Silicon micro-pillars coated with DLC & 145 & - & 0.12 & [29] \\
\hline Silicon micro-pillars coated with ZDOL & 105 & - & 0.08 & [29] \\
\hline SU-8 micro-patterns & 105 & - & 0.3 & [30] \\
\hline SU-8 micro-patterns coated with PFPE & 108 & - & 0.19 & [30] \\
\hline SU-8 micro-patterns treated with Ar and coated with PFPE & 83 & - & 0.21 & [30] \\
\hline SU-8 micro-patterns treated with $\mathrm{O}_{2}$ and coated with PFPE & 91 & - & 0.22 & [30] \\
\hline
\end{tabular}

In these works, atomic force microscope and micro-tribotesters were used to measure friction at nano- and micro-scales, respectively.

various biomimetic patterned surfaces mimicking the protuberances of lotus leaves, and have achieved significant reduction in surface forces at micro/nano-scale, which are described below.

\section{Biomimetic nano-patterned surfaces}

Polymers provide a fertile ground for biomimetic approaches as they offer a wide range of materials and easy fabrication routes capable of creating surfaces with controllable properties. In an earlier work, nano-scale polymer patterns resembling the protuberances on lotus leaves were created on poly(methyl methacrylate) (PMMA) films spin-coated on silicon surfaces, using capillary force lithography (CFL) [13]. Figure 2C shows a scanning electron microscope (SEM) image of the patterns fabricated at a holding time of $60 \mathrm{~min}$. Such nano-patterns are hydrophobic when compared to silicon surface. Moreover, approximate calculation of contact area based on simple geometry reveals that the contact area can be lowered by 7 times in the case of nano-patterns as compared to silicon flat surface. By the virtue of their hydrophobicity and the capability to reduce contact area substantially, the nano-patterns reduced friction force by 14-24 times at nano-scale, when the friction force was measured using an atomic force microscope [13]. In these measurements, when the applied normal load was kept zero, friction force in silicon was measured to be $20 \mathrm{nN}$, whereas for nanopatterns it was $\sim 0.84 \mathrm{nN}$. It is worth noting that the friction force exists even at the zero applied normal load, at nano-scale. This is due to the influence of intrinsic adhesion on friction force [13]. The large decrease in friction force induced by the nano-patterns in the absence/
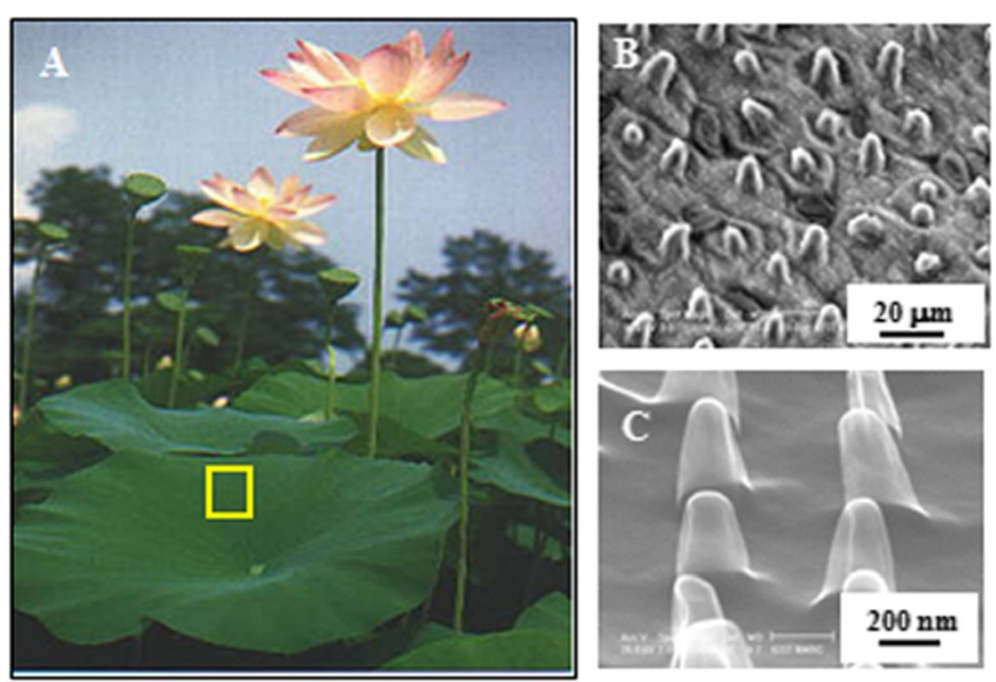

Figure 2 (A) Lotus plant, (B) micron-sized protuberances on lotus leaf and (C) biomimetic nano-patterns [13]. 
presence of an applied normal load is indicative of the ability of the patterned surfaces to significantly reduce inherent adhesion and friction.

In general, contact area plays an important role in defining friction at micro/nano-scale. Unlike at macroscale where friction is independent of contact area between surfaces (Amonton's law, [4,14]), at small-scales friction is strongly dependent on contact area $[4,14,15]$. According to Bowden and Tabor, friction force directly depends on the real area of contact for a single asperity contact $\left(F_{f}=\tau A_{v}\right.$ where $\tau$ is the shear strength, an interfacial property, and $A_{r}$ the real area of contact [16]). This law is effective at micro/nano-scale and any reduction in real area of contact directly reduces friction between surfaces at these scales. As a result, patterned surfaces directly reduce real area of contact as similar to the protuberances on lotus leaves.

Patterning of surfaces causes a reduction in real area of contact when the size of the patterns is considerably smaller than that of the counterface [17] (e.g. ball, Figure 3). Figure 3 illustrates the reduction of contact area in patterned surfaces. If the contact area between a ball and a flat surface is A ( $\pi \mathrm{a}^{2}$, where ' $\mathrm{a}$ ' is the contact radius given by contact mechanics models [18]), the contact area between the ball and the patterned surface is lower than $\mathrm{A}$, as the contact between them occurs only at the tip of the asperities/pillars (indicated by arrows). Figure 4A shows the basic parameters involved in the design of patterned surfaces, e.g., the diameter (d) and height (h) of each asperity/pillar and pitch (p), the distance between any two adjacent asperities/pillars. In designing patterned surfaces, these parameters should be suitably selected so as to ensure that: (i) reduction in friction occurs via the reduction in contact area and (ii) they have good load bearing capacity. Depending on the

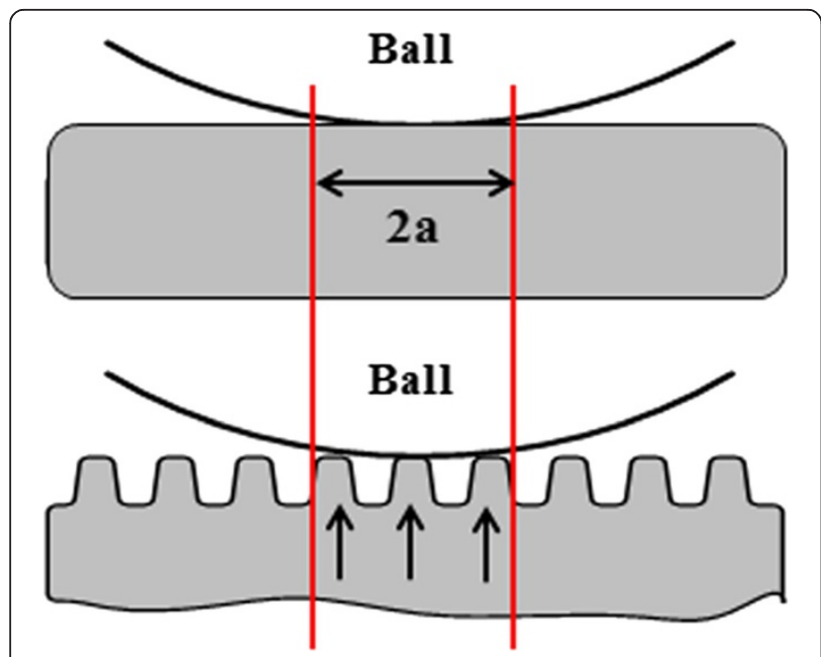

Figure 3 Schematic of counterface balls of the same size making contact with a flat surface and a patterned surface. size of the counterface (e.g. radius of curvature of the counterface ball, Figure 3), pitch can be varied accordingly. Increase in pitch decreases the contact area, i.e. a patterned surface with a larger pitch gives rise to a lower contact area than that with a smaller pitch, thereby reducing the friction even further (Figure 4, [19]).

To investigate the capability of biomimetic patterned surfaces, CFL has been extensively used to create polymer patterns with varying sizes and shapes in a simple and cost effective manner [20]. This soft lithography technique utilizes the competition between capillary and hydrodynamic forces in the course of pattern formation. Consequently, variation in size and shape of patterns can be achieved by adjusting the holding temperature and time via various dynamic motions of the polymer (e.g., meniscus rise and polymer reflow) [21]. Figure 5 shows some examples of nano-patterns with varying shapes and sizes fabricated by CFL [13,21]. At the holding temperature of $120^{\circ} \mathrm{C}$ and time of $15 \mathrm{~min}$, dimpled patterns initially formed as a result of meniscus formation, which evolved to dimpled pillars with the increase of process time to 30 min (Figure 5A-B). When the holding temperature was raised to $150^{\circ} \mathrm{C}$ and maintained for $15 \mathrm{~min}$, spherical patterns formed and further transformed into conical pillars with the increase of time to 30 min (Figure 5C-D). Friction of these nano-patterns has been found to be much lower than that of the flat surface, being dependent on their size and shape [13,21]. The friction of nano-patterns can be further reduced by applying hydrophobic films such as self-assembled monolayers [22].

Similar to polymer nano-patterns, DLC nano-dots on silicon surfaces were also used to reduce friction at micro/nano-scale [23]. The nano-dots were created by depositing DLC films on randomly distributed nano-sized nickel $(\mathrm{Ni})$ dots that had been synthesized on silicon surfaces by annealing Ni thin films (Figure 6A-B) [24]. These surfaces demonstrated a reduction in friction force by 17-20 times at nano-scale, and a reduction of friction coefficient $(\mu)$ by 3.5 times at micro-scale, when compared to those of silicon flat surface. It was claimed that the hydrophobicity of the nano-dot surfaces and their reduced contact area brought about such a collective reduction in the friction properties. Micro/nano-textured surfaces such as the ones shown in Figure $6 \mathrm{C}$-D, also prove beneficial in reducing adhesion and friction forces [17].

\section{Biomimetic micro-patterned surfaces}

At micro-scale, although polymer nano-patterns (Figure 2C) reduce $\mu$ value by 1.4 times compared to that of silicon, they undergo plastic deformation and become ineffective within a short duration of time [13], suggesting that nano-patterns have limited load bearing capacity at micro-scale. Therefore, micro-patterns would be better 


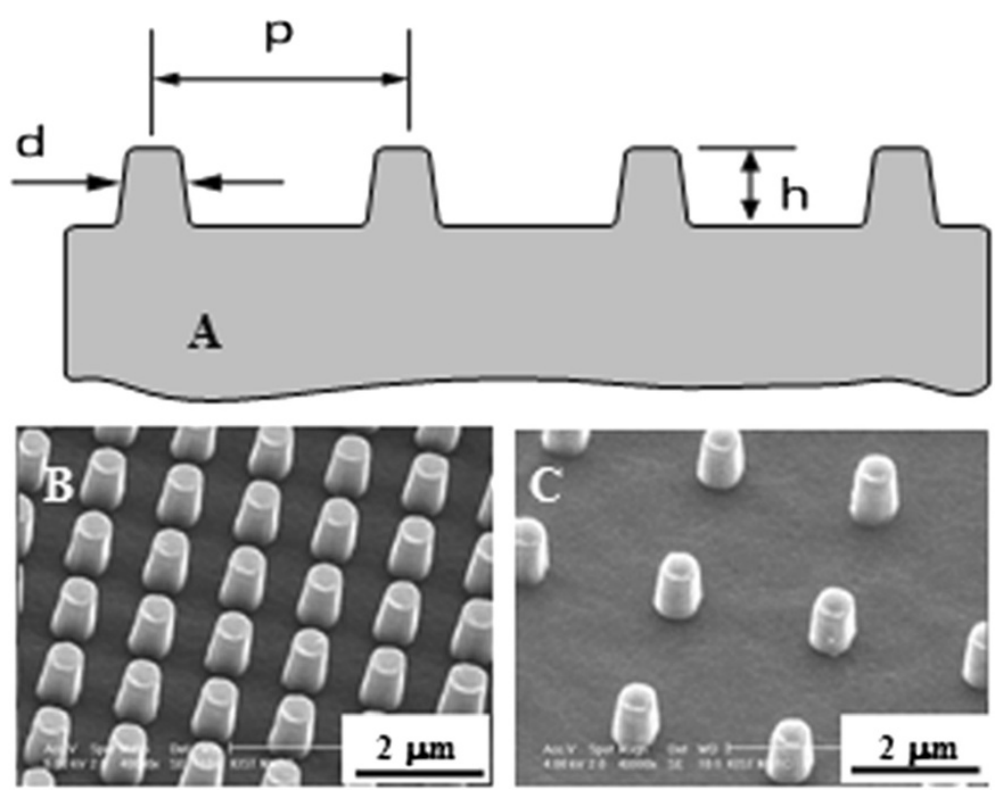

Figure 4 (A) Schematic of a patterned surface showing the basic design parameters, (B) polymer patterns with small pitch and (C) polymer patterns with large pitch.

suited for micro-scale. A simple, direct way of fabricating micro-patterns is to replicate natural surfaces of water repellent plants (e.g., lotus and colocasia) with a polymer $[25,26]$. It is noted in this regard that similar to lotus, colocasia also has protuberances with micron-sized wax crystals, making its leaves water-repellent (water contact angle: $164^{\circ}[10]$ ). Figure 7 shows SEM images of replicated surfaces of real lotus and colocasia leaves by CFL on PMMA films spin-coated on silicon substrates. For the replication, real leaf surfaces were used as templates.
These replicated surfaces were hydrophobic and showed $\mu$ values 3.5-4 times lower than that of silicon surfaces. the replicated surfaces were more durable than their nano-scale counterparts (nano-patterns, Figure 2C [13]), at microscale.

Given the fact that the replicated surfaces have asperities larger in size $(\mu \mathrm{m})$ than that of nano-patterns $(\mathrm{nm})$, it could be anticipated that their $\mu$ values would be comparatively higher than those of the nano-patterns as a result of higher contact area. However, two distinct and
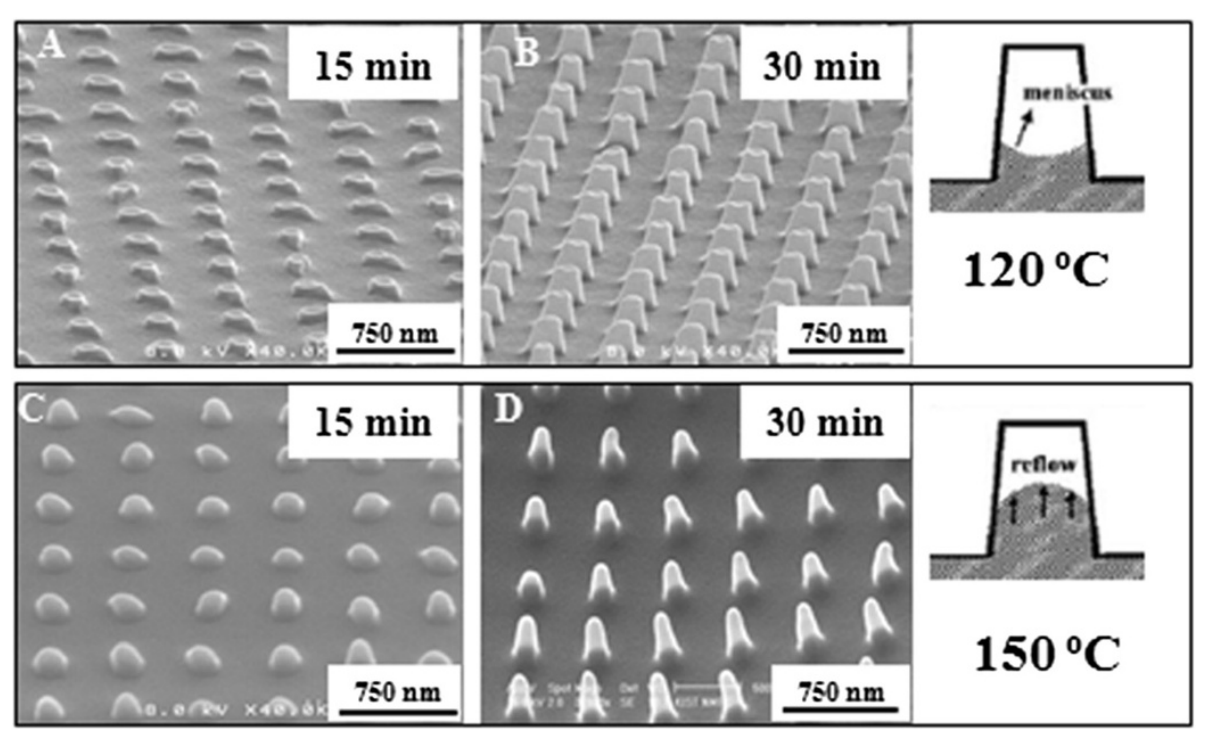

Figure 5 Polymer nano-patterns with different size and shape created using CFL by varying the holding temperature and time [13,21]. 

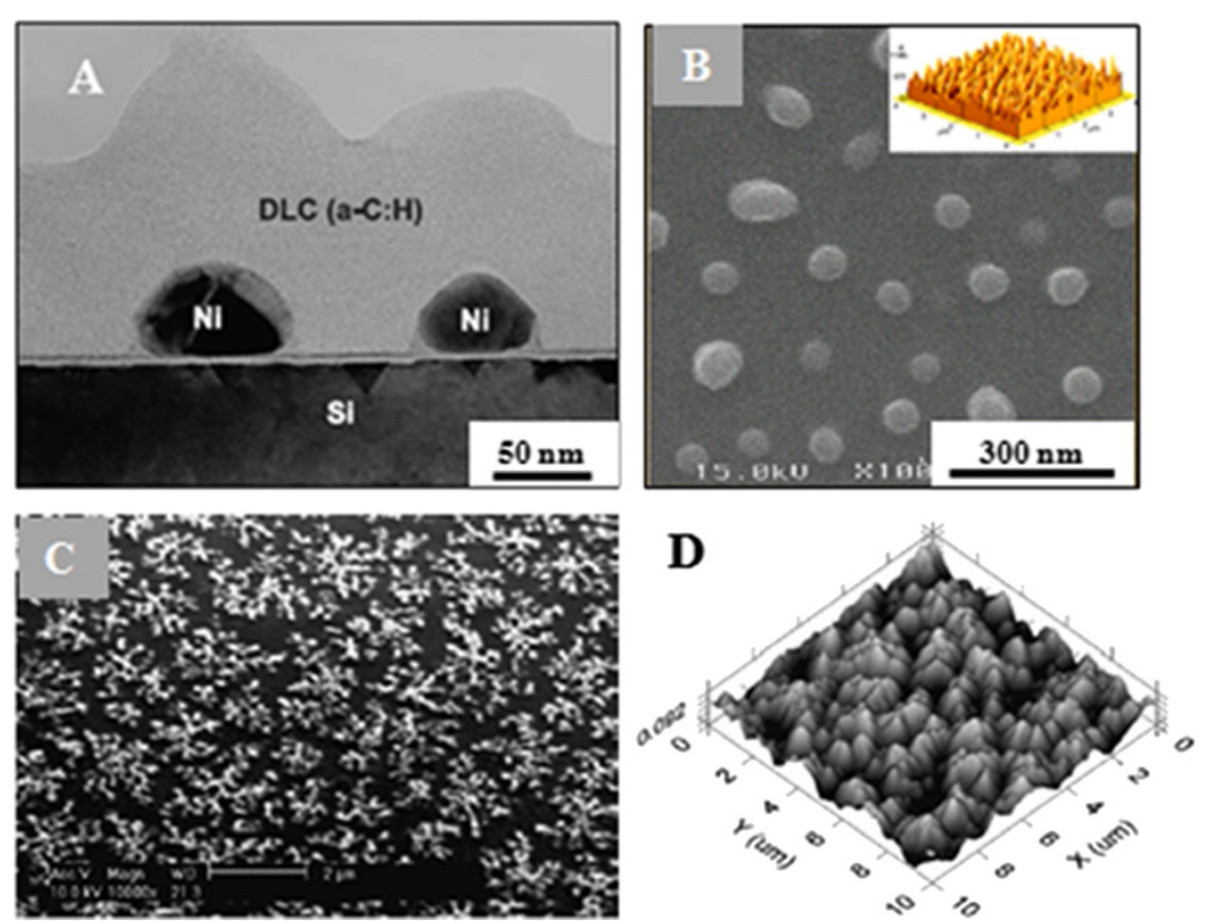

Figure 6 (A \& B) DLC layer on nano-sized Ni dots synthesized on silicon surface [23,24], (C \& D) micro/nano-textured surface produced by UV assisted crystallization of amorphous Si [17].

remarkable features of the replicated surfaces come into play in lowering the contact area, leading to lower $\mu$ values: (i) the distance between adjacent asperities is on a larger scale when compared to that of the nanopatterns and (ii) only those asperities that are high enough come in contact with the counter surface. In conjunction with these attributes, confocal microscope images of the replicated surfaces demonstrate that in a given nominal area, not all asperities within the area have uniform height (Figure 8). Only those asperities that are high enough make contact with the counterface ball. In contrast, in the case of nano-patterns all asperities are
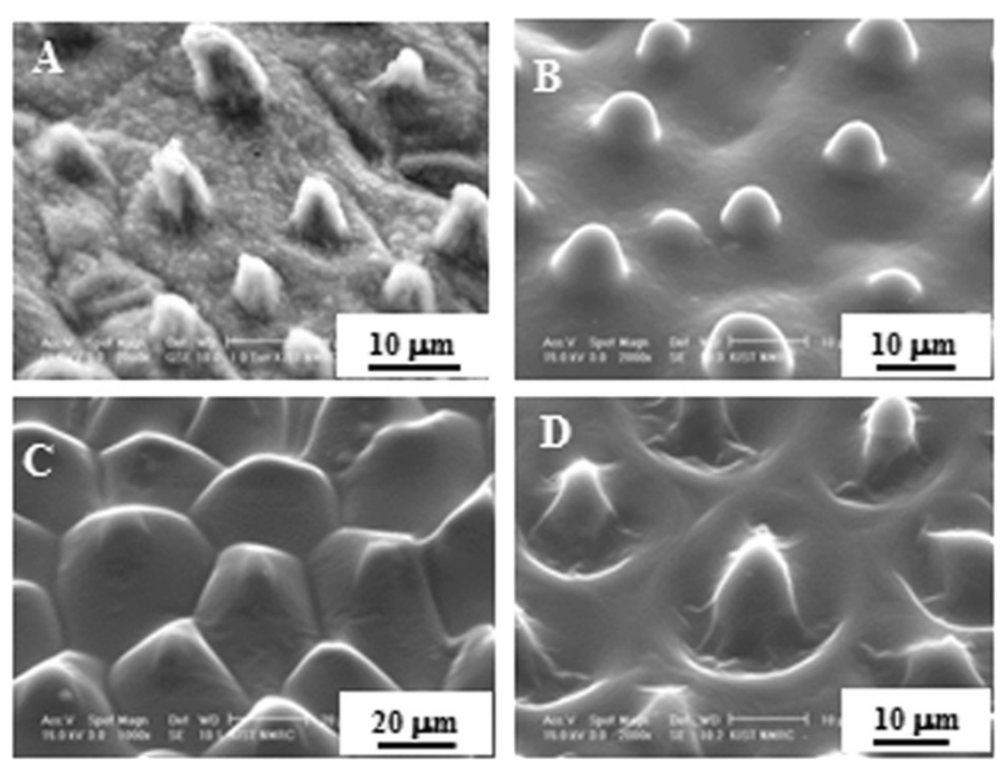

Figure 7 (A) Real lotus leaf surface and (B)-(D) replicated surfaces of real leaves of lotus, colocasia and colocasia (dry), respectively [25,26]. 

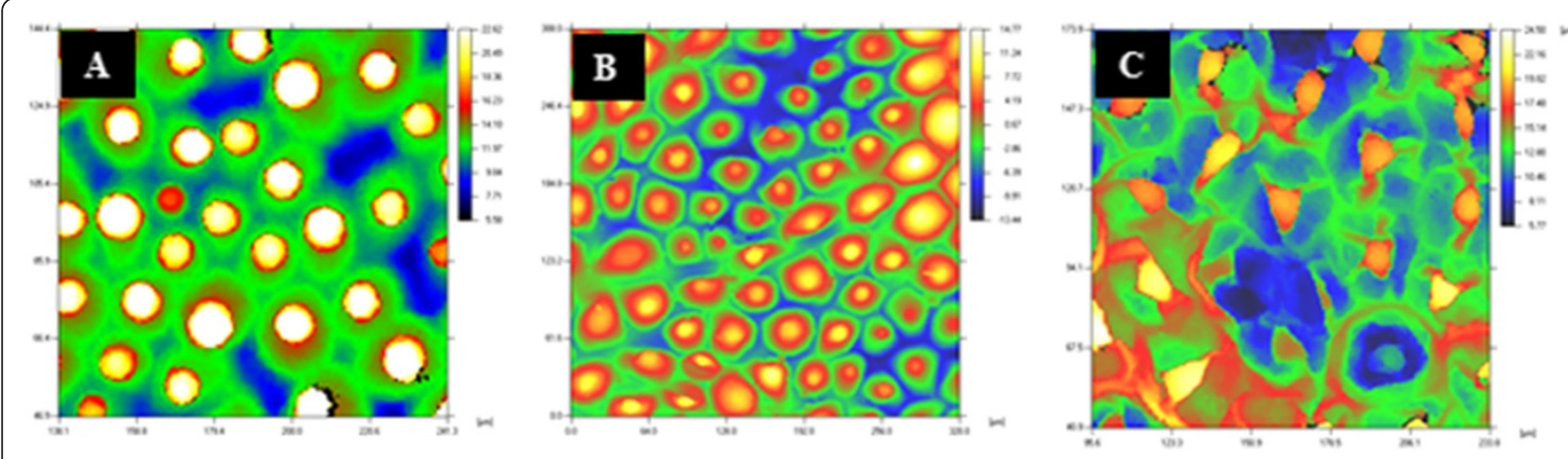

Figure 8 Confocal microscope images: (A)-(C) replicated surfaces of real leaves of lotus, colocasia and colocasia (dry), respectively.

of the same height and those that are present within a nominal contact area invariably make contact with the counterface ball.

Taken together, the large scale inter-asperity distance and uneven heights of the asperities in the replicated surfaces retain the $\mu$ value at lower levels for the micropatterns. Further, it is found that the surface topography of replicated surfaces follows the real leaves on a smoother scale (Figure 7), even though the real leaves have hierarchical roughness due to the presence of micro-scale protuberances and nano-scale wax on them. These findings have important implications towards controllable friction with topographically patterned surfaces. Specifically, a simple replication of the surface structures of natural water-repellent leaves would suffice in order to decrease micro-frictional property via the reduction in contact area. If one wants to reproduce the detailed surface topography of real leaves with high accuracy, it would be difficult, costly, and time-consuming, and further may not contribute to lower friction force at small scale.

To explain this further, micro- and nano-scale roughness on surfaces, i.e. dual-scale hierarchical surfaces provides superhydrophobicity (e.g. Figure 9A [27]). But, in the context of reducing friction, the additional nano-scale roughness on micro-patterns does not prove to be really effective. This is clearly evident from an investigation on replication of lotus leaf surfaces, with/without the addition of nano-scale features [28]. In this work, lotus leaf surfaces were replicated using an acetate film, followed by electrodeposition of nanocrystalline Ni (Figure 9B) [28]. Ni crowns were created on top of the replicated microprotuberances by short-duration electrodeposition of $\mathrm{Ni}$. A PFPE (perfluoropolyether) solution treatment of the surfaces gave rise to a nanotextured layer on $\mathrm{Ni}$ crowns that were present on the protuberances (inset, Figure 9B). Water contact angle on replicated protuberances increased from $91^{\circ}$ to $156^{\circ}$ when the nanotextured layer was added to the replicated surfaces [28]. It was reported that the nano-scale surface texture on the protuberances, which played an important role in increasing the hydrophobicity was not useful in lowering the friction coefficient of the replicated surfaces any further [28]. From this result it can be understood that accurate replication of hierarchical roughness of real leaves is not necessary in order to reduce friction, rather replication of the protuberances on micro-scale will suffice.

\section{Combination of nanolubricant and micro-patterned surfaces}

Apart from the synthetic lotus-like surfaces, microfriction experiments conducted directly on real lotus leaf surfaces have shown that the wax on their surface can
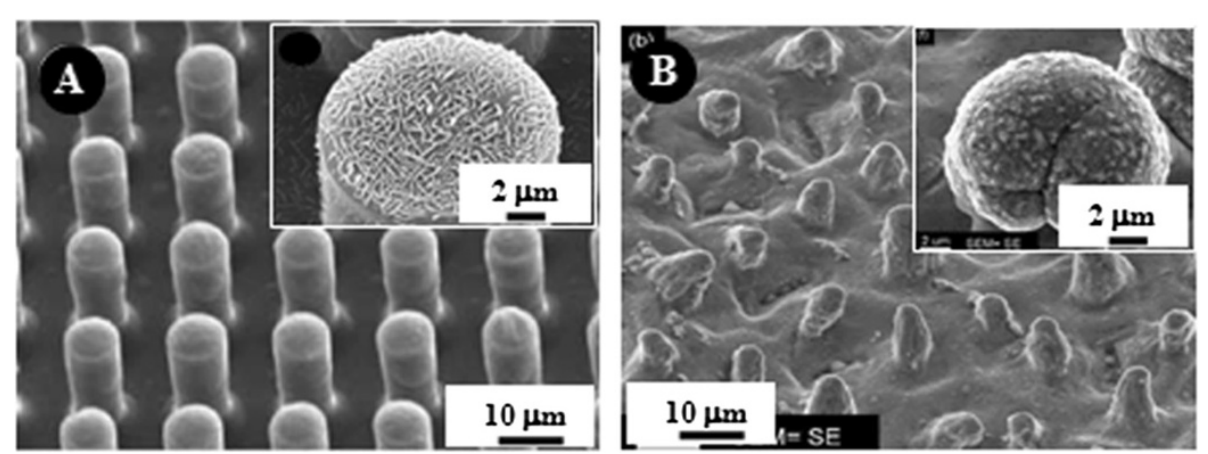

Figure 9 (A) Hierarchical superhydrophobic surface (platelets on a micro-pillar) [27] and (B) replicated surface of lotus leaf [28]. 
act as a lubricant with reduced friction [29]. The $\mu$ value of the lotus leaves in their fresh condition was significantly lower $(\sim 0.01)$ than that in their dried conditions $(\sim 0.15)$, which was attributed to the presence of wax in its fresh condition [29]. Taking a cue from this observation, an application of boundary lubricants such as DLC and ZDOL (dihydroxy derivative of perfluoropolyoxyalkane) on silicon micro-pillars (Figure 10) has proven to be effective in increasing the hydrophobicity and surface lubricity, resulting in lower $\mu$ values [29]. Micro-pillars fabricated on silicon wafers by photolithography were seen to reduce friction by 2.8 times compared to that of silicon flat surface. However, they underwent wear owing to the brittle nature of silicon as well due to increased contact pressure (at any given load, patterned surfaces experience higher contact pressures than flat surfaces due to lower contact areas). Upon applying DLC and ZDOL lubricants to the micro-pillars, the $\mu$ value dropped by 1.3-2 times that of the bare micro-pillars because of the lubrication effect [29]. No wear/material removal was observed on the lubricated micro-pillars that exhibited $\mu$ values of 3.8-5.8 times lesser than that of silicon flat surface [29].

In order to achieve low friction and delay the onset of plastic deformation/wear of polymer patterns, chemical anchoring/grafting of nanolubricant molecules onto micro-patterned surfaces is necessary. A recent biomimetic work reporting on the reduced friction for SU-8 polymer supports this argument [30]. In this work, micro-patterns in the form of micro-bumps were fabricated by nano-imprint lithography on SU-8 thin films spin-coated on silicon surfaces. Results showed that the micro-patterns exhibited $\mu$ values 2 times lower than that of SU-8 thin film, but underwent plastic deformation in short time duration of $\mathrm{t}<0.5 \mathrm{~min}$ (100 cycles).

Surface modifications were conducted to the SU-8 micro-patterns by coating perfluoropolyether (PFPE) nanolubricant in two different ways: (i) direct coating and (ii) coating after their separate exposure to argon (Ar) and oxygen $\left(\mathrm{O}_{2}\right)$ plasmas. The modified micro-patterns showed a decrease in $\mu$ value by $>1.4$ times in comparison to that of un-modified micro-patterns, and by $>2.7$ times than that of SU-8 thin film. No plastic deformation/ wear was observed on the modified micro-patterns when tested for the time duration of $\mathrm{t}>8$ hours $(100,000$ cycles), at $0.3 \mathrm{~N}$ applied normal load and $15 \mathrm{~mm} / \mathrm{s}$ sliding speed. The modified micro-patterns exhibited enhanced durability by $>1000$ times when compared to that of un-modified micro-patterns and polymer thin film. With an increase in load and speed to $1 \mathrm{~N}$ and $42 \mathrm{~mm} / \mathrm{s}$, although the modified micro-patterns showed low $\mu$ values $(\leq 0.15)$, clear difference was observed in their durability responses. In detail, micro-patterns directly coated with PFPE had durability of $14 \mathrm{~min}$, whereas those pre-treated with $\mathrm{Ar}$ and $\mathrm{O}_{2}$ plasmas before being coated with PFPE had durability of $32 \mathrm{~min}$ and $60 \mathrm{~min}$, respectively. This is because Ar plasma generates carbonyl groups $(\mathrm{C}-\mathrm{O}$ and C-O-O groups), while $\mathrm{O}_{2}$ plasma carboxyl groups $(\mathrm{COOH}$ groups), each altering the surface properties differently. Thus, the plasma-treated micro-patterns have polar functional groups on their surfaces, to which the hydroxyl groups of PFPE nanolubricant at both their terminal ends get strongly bound, promoting the durability of micropatterns.

It needs to be further emphasized that the difference in the observed durability amongst the plasma-treated micro-patterns coated with PFPE is attributed to the type of bonding that occurs between PFPE molecules and chemically activated micro-patterned surfaces. In general, hydroxyl groups form covalent bonds with carboxyl groups, whereas with carbonyl groups they form hydrogen bonds. Covalent bonds are stronger than hydrogen bonds and thus when nanolubricant molecules are covalently bonded onto micro-patterned surfaces, they exhibit significant enhancement in their durability in terms of wear resistance capacity. Hence, it was observed that the micro-patterns treated with $\mathrm{O}_{2}$ plasma followed by coating with PFPE showed higher durability

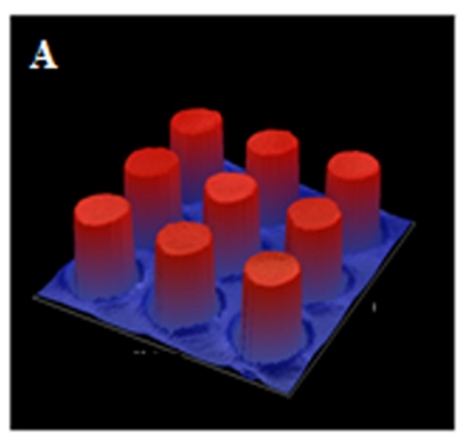

B

DLC/ZDOL Overcoat

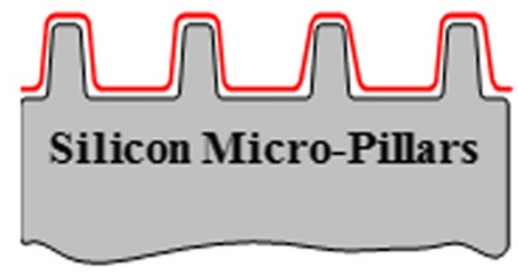

Figure 10 Schematic of: (A) biomimetic silicon micro-pillars fabricated by photolithography [29] and (B) boundary lubricant overcoats on the biomimetic silicon micro-pillars. 
when compared to those treated with Ar plasma followed by PFPE coating.

Based on the numerous research outcomes mentioned until now, polymeric micro-patterns with low friction and enhanced durability would be the best candidates for small-scale devices. Also, in other applications such as optoelectronics and photonics, polymer patterns are increasingly employed [31] for engineering reliable mechanical motion.

\section{Discussion}

As described earlier, the area-to-volume ratio is extremely large at micro/nano-scale, leading us to conclude that friction depends on surface properties rather than bulk material properties. On a comparative note, the difference between thin films/coatings and biomimetic patterned surfaces lies in their approach to reduce/ control friction. While the former is based on chemical modification of surfaces, the latter relies on topographi$\mathrm{cal} /$ physical modification.

Contact area in the case of thin films/coatings can be estimated by using the Johnson-Kendall-Roberts (JKR) contact mechanics model [32]: $A_{r}=\pi\left[R / K\left(F_{n}+6 \pi \gamma g R+\right.\right.$ $\left.\left.\left[12 \pi \gamma R F_{n}+(6 \pi \gamma R)^{2}\right]^{1 / 2}\right)\right]^{2 / 3}$. Here, $R$ is the size of the counterface ball, $\mathrm{K}$ the effective elastic modulus (combination of elastic modulus of coating and counterface), $F_{n}$ the applied normal load and $\gamma$ the interfacial energy. For thin films/coatings, SAMs have low elastic modulus [33] and so the reduction in contact area is not significant. To reduce friction, a complex chemistry has to work such that the suitable selections of end groups, chain length and mixture of monolayers have to be taken into account [8]. Further, achieving uniform coating of SAMs on large surface areas without defects or polymerization poses a great challenge. On the other hand, in DLC coatings, elastic modulus is relatively high so that reduction in contact area plays a supportive role in reducing friction [15]. It is noted that lubrication in DLC coatings mainly occurs by the formation of 'transfer layer' [34]. During the formation of transfer layer, not all the material that gets removed from DLC coating is transferred to the counter surface; rather most of it gets released in the form of loose wear particles. Such free wear particles could play an adverse role by interfering physically and/or chemically with the functioning components (DLC has high hardness, electrical insulation, thermal conduction and chemical inertness [35]). Regarding vapor phase lubrication method [4], it is necessary to ensure the presence of lubricant vapor at the interfaces for the entire duration time of device operation. Given this situation, it requires a provision to store lubricant vapor within the device and continuously re-supply it to the interfaces. This remains a challenging issue yet to be addressed.
Given the above-mentioned limitations/drawbacks of thin films/coatings and vapor phase lubrication, biomimetic patterned surfaces have some noteworthy salient features: (i) they provide a simple and easy route to reduce friction via the direct reduction in contact area. In addition, their performance can be further enhanced by applying boundary lubricant thin films/coatings. (ii) Lithography techniques used to make patterns are the same as those used to fabricate MEMS/NEMS, indicating that patterning the surfaces of device components can be easily conducted during their fabrication. This makes the process even more convenient and cost- and time-effective compared to other methods. (iii) The main aim to reduce friction in small-scale devices is not to eliminate friction completely, since with zero friction sliding components cannot engage each other. Instead, friction should be controlled in such a way that it should be low enough to support smooth motion, but sufficiently high to engage relative mechanical motion between components, e.g. micro-gears. Taken together, making patterns on component surfaces with varying sizes and shapes along with the application of a variety of boundary lubricants provides greater flexibility and strength to control friction at micro/nano-scale, for desired smooth operations of MEMS/NEMS.

Considering the theoretical modelling of biomimetic surfaces, it is focused towards understanding the effect of hierarchical roughness on adhesion force [36-40], especially 'roughness induced superhydrophobicity'. The studies aim to support the design of low-adhesion, lowfriction and non-wetting surfaces. Wetting involves interactions at different scale levels/sizes, namely macroscale (water drop), micro-scale (surface texture) and nanoscale (molecules). Hence, understanding the role of hierarchical surface roughness, the transition between various wetting regimes and optimization of roughness etc., becomes important. Modelling has given some insights on solid-liquid interactions. As an example, studies on wetting of micro-patterned surfaces have shown that: (i) the wetting behavior depends on the spacing factor (a micro-scale non-dimensional parameter), and (ii) the contact angle hysteresis and Cassie-Wenzel transition are governed by micro- and nano-scale effects. These insights on the fundamental mechanisms of wetting are very useful in designing non-adhesive surfaces. It is to be noted that when compared to solid-liquid adhesion, dry solid-solid friction is a complex phenomenon that at times is influenced by wear, and so is rather difficult to model.

\section{Conclusions}

We have presented that the underlying principles in natural surfaces provide valuable insights towards solving intricate technological issues. Biomimetic engineering of surfaces is an exciting and practical way to control friction 
at small-scales. Examples presented in this review clearly indicate that by the design and development of biomimetic patterned surfaces, in combination with boundary lubrication, better solutions can be pursued to control friction at small-scales, for the efficient operation and application of miniaturized devices.

\section{Competing interests}

The authors declare that they have no competing interests.

\section{Authors' contributions}

AS wrote the manuscript and KYS edited. Final manuscript has been read and approved by both the authors.

\section{Authors' information}

Arvind Singh received his doctoral degree from the Indian Institute of Science (IISC), India in 2003. In his doctoral research work, he consolidated a new approach to understand metallic wear based on dynamically evolved microstructure. As a Materials Scientist at the John F. Welch Technology Center (GE India), he investigated tribological systems in aircraft engines and on super-abrasives. During his tenure as a Visiting Scientist at the Korea Institute of Science and Technology (KIST), South Korea, he proposed micro/ nano-patterns as biomimetic surfaces for tribological application in MEMS/ NEMS. He continued his research on biomimetics at the National University of Singapore (NUS), where he formulated novel surface modification methods to functionalize SU-8 polymer for tribological applications in MEMS/Bio-MEMS and for indwelling biomedical devices. At Vestas Global $R \& D$, he investigated tribology of wind energy systems. Currently, at the Energy Research Institute (ERIAN), Singapore, he is leading tribology and surface engineering R\&D projects related to wind \& marine renewables. Kahp-Yang Suh received his doctoral degree from the Seoul National University (SNU), Korea in 2002. He specialized in micro/nano-patterning of polymeric surfaces using capillary force lithography/nanoimprint lithography, and made significant contributions to the field. He was a postdoctoral researcher at the Massachusetts Institute of Technology (MIT), USA. In 2004 he joined as an Assistant Professor at SNU. He was an outstanding faculty and researcher in the field of fabrication of micro/nano-polymeric patterns, whose applications ranged from tribology to lab-on-chip devices. He won a number of awards, including TR100 Young Innovator Award by MIT Technology Review, Best Graduate Student Award by the Brain Korea (BK) 21 Program of the Korean Government, Korea Presidential Young Scientist Award and Young Professor Award by College of Engineering of SNU. Arvind Singh and Kahp-Yang Suh, shared the 'First Surface Engineering Best Paper Award', given by the Society of Tribologists \& Lubrication Engineers (STLE), USA (2007), for their pioneering work on biomimetic approach as a robust solution for tribological issues in micro/nano-scale devices.

\section{Acknowledgment}

Arvind Singh dedicates this review article to Kahp-Yang Suh, for his memorable contribution to the field.

\section{Author details}

'Energy Research Institute (ERIAN), Clean Tech One, Nanyang Technological University, Singapore 637141, Singapore. ${ }^{2}$ Department of Mechanical and Aerospace Engineering, Seoul National University, Seoul 151-742, South Korea.

\section{Received: 21 September 2013 Accepted: 6 December 2013} Published: 18 December 2013

\section{References}

1. Adams TM, Layton RA (2010) Introductory MEMS: Fabrication and Applications. Springer, New York

2. Tanner DM, Smith NF, Irwin LW, Eaton WP, Helgesen KS, Clement JJ, Miller MW, Walraven JA, Peterson KA, Tangyunyong P, Dugger MT, Miller SL (2000) MEMS Reliability: Infrastructure, Test Structures, Experiments, and Failure Modes. In: Sandia Report, SAND2000-0091, Sandia National Laboratories

3. de Boer MP, Mayer TM (2001) Tribology of MEMS. MRS Bull 26:302-304

4. Kim SH, Asay DB, Dugger MT (2007) Nanotribology and MEMS. Nano Today 2:22-29
5. MEMS [http://www.microchem.com/Appl-MEMs-SurfaceMod.htm]

6. Carpick RW (2006) Controlling friction. Science 313:184-185

7. Singh RA, Satyanarayana N, Sinha SK (2011) Surface chemical modification for exceptional wear life of MEMS materials. AIP Adv 1:042141

8. Tambe NS, Bhushan B (2005) Nanotribological characterization of self-assembled monolayers deposited on silicon and aluminium substrates. Nanotechnology 16:1549-1558

9. Grierson DS, Carpick RW (2007) Nanotribology of carbon-based materials. Nano Today 2:12-21

10. Neinhuis C, Barthlott W (1997) Characterization and distribution of water-repellent, self-cleaning plant surfaces. Ann Bot 79:667-677

11. Zorba V, Stratakis E, Barberoglou M, Spanakis E, Tzanetakis P, Anastasiadis SH, Fotakis C (2008) Biomimetic artificial surfaces quantitatively reproduce the water repellency of a lotus leaf. Adv Mater 20:4049-4054

12. Gould P (2003) Smart, clean surfaces. Mater Today 6:44-48

13. Yoon E-S, Singh RA, Kong H, Kim DH, Jeong HE, Suh KY (2006) Tribological properties of bio-mimetic nano-patterned polymeric surfaces on silicon wafer. Tribol Lett 21:31-37

14. Mo Y, Turner KT, Szlufarska I (2009) Friction laws at the nanoscale. Nature 457:1116-1119

15. Yoon E-S, Singh RA, Oh HJ, Kong H (2005) The effect of contact area on nano/micro-scale friction. Wear 259:1424-1431

16. Bowden FP, Tabor D (1950) The Friction and Lubrication of Solids. Clarendon Press, Oxford

17. Zou M, Cai L, Wang H, Yang D, Wyrobek T (2005) Adhesion and friction studies of a selectively micro/nano-textured surface produced by UV assisted crystallization of amorphous silicon. Tribol Lett 20:43-52

18. Scherge M, Gorb SN (2001) Physical principles of micro- and nanotribology. In: Biological micro- and nanotribology. Springer, Berlin Heidelberg

19. Pham DC, Na K, Yang S, Kim J, Yoon ES (2009) Microtribological properties of topographically-modified polymeric surfaces with different pitches. J Korean Phys Soc 55:1416-1424

20. Suh KY, Kim YS, Lee HH (2001) Capillary force lithography. Adv Mater 13:1386-1389

21. Suh KY, Jeong HE, Kim DH, Singh RA, Yoon E-S (2006) Capillarity-assisted fabrication of nanostructures using a less permeable mold for nanotribological applications. J Appl Phys 100:034303

22. Burton Z, Bhushan B (2005) Hydrophobicity, adhesion, and friction properties of nanopatterned polymers and scale dependence for micro- and nanoelectromechanical systems. Nano Lett 5:1607-1613

23. Singh RA, Na K, Yi JY, Lee KR, Yoon E-S (2011) DLC Nano-dot surfaces for MEMS Tribology. Appl Surf Sci 257:3153-3157

24. Park SJ, Lee KW, Ko DH (2005) Tribological behavior of nano-undulated surface of diamond-like carbon films. Diam Relat Mater 14:1291-1296

25. Singh RA, Yoon E-S, Kim HJ, Kong H, Park S, Jeong HE, Suh KY (2007) Enhanced tribological properties of lotus leaf-like surfaces fabricated by capillary force lithography. Surface Eng 23:161-164

26. Singh RA, Yoon E-S, Kim HJ, Kim J, Jeong HE, Suh KY (2007) Replication of surfaces of natural leaves for enhanced micro-scale tribological property. Mat Sci Eng C-Bio S 27:875-879

27. Bhushan B, Jung YC, Koch K (2009) Micro-, nano- and hierarchical structures for superhydrophobicity, self-cleaning and low adhesion. Phil Trans R Soc A 367:1631-1672

28. Shafiei M, Alpas AT (2009) Nanocrystalline nickel films with lotus leaf texture for superhydrophobic and low friction surfaces. Appl Surf Sci 256:710-719

29. Singh RA, Pham DC, Kim J, Yang S, Yoon E-S (2009) Bio-inspired dual surface modification to improve tribological properties at small-scale. Appl Surf Sci 255:4821-4828

30. Singh RA, Siyuan L, Satyanarayana N, Kustandi TS, Sinha SK (2011) Bio-inspired polymeric patterns with enhanced wear durability for microsystems applications. Mat Sci Eng C-Biomim 31:1577-1583

31. Park H, Cheng X (2009) Thermoplastic polymer patterning without residual layer by advanced nanoimprinting schemes. Nanotechnology 20:245308

32. Johnson KL, Kendall K, Roberts AD (1971) Surface energy and contact of elastic solid. Proc R Soc A 324:301-313

33. DelRio FW, Jaye C, Fischer DA, Cook RF (2009) Elastic and adhesive properties of alkanethiol self assembled monolayers on gold. Appl Phys Lett 94:131909

34. Williams JA, Le HR (2006) Tribology and MEMS. J Phys D Appl Phys 39:201-214 
35. Mattox DM (1998) Handbook of Physical Vapor Deposition (PVD) Processing. Elsevier, New York

36. Nosonovsky M, Bhushan B (2008) Biologically inspired surfaces: broadening the scope of roughness. Adv Funct Mater 18:843-855

37. Nosonovsky M, Bhushan B (2008) Patterned nonadhesive surfaces: superhydrophobicity and wetting regime transitions. Langmuir 24:1525-1533

38. Nosonovsky M, Bhushan B (2008) Energy transitions in superhydrophobicity: low adhesion, easy flow and bouncing. J Phys Condens Matter 20:395005-395011

39. Nosonovsky M, Bhushan B (2007) Biomimetic superhydrophobic surfaces: multiscale approach. Nano Letts 7:2633-2637

40. Nosonovsky M, Bhushan B (2007) Multiscale friction mechanisms and hierarchical surfaces in nano- and bio-tribology. Mat Sci Eng R 58:162-193

doi:10.1186/2213-9621-1-6

Cite this article as: Singh and Suh: Biomimetic patterned surfaces for controllable friction in micro- and nanoscale devices. Micro and Nano Systems Letters 2013 1:6.

\section{Submit your manuscript to a SpringerOpen ${ }^{\circ}$} journal and benefit from:

- Convenient online submission

- Rigorous peer review

- Immediate publication on acceptance

- Open access: articles freely available online

- High visibility within the field

- Retaining the copyright to your article 